\title{
Photonic chip based tunable slow and fast light via stimulated Brillouin scattering \\ Ravi Pant ${ }^{(1)}$, Adam Byrnes ${ }^{(1)}$, Christopher G. Poulton ${ }^{(2)}$, Enbang Li ${ }^{(1)}$, Duk-Yong Choi ${ }^{(3)}$, Steve Madden ${ }^{(3)}$, Barry Luther-Davies ${ }^{(3)}$, and Benjamin J. Eggleton ${ }^{(1)}$ \\ ${ }^{(1)}$ Centre for Ultrahigh bandwidth Devices for Optical Systems (CUDOS), Institute of Photonics and Optical Science, School of Physics, The University of Sydney, NSW 2006, Australia. \\ (2) CUDOS, University of Technology Sydney, Sydney, NSW 2007, Australia. (3) CUDOS, Laser Physics Centre, Australian National University, Canberra, ACT 0200,
} Australia

\begin{abstract}
The ability to control the speed of light on an optical chip is fundamental to the development of nanophotonic components for alloptical signal processing and sensing [1-7]. However this is a significant challenge, because chip-scale waveguides require very large changes in group index $\left(\Delta \mathrm{n}_{\mathrm{g}}\right)$ to achieve appreciable pulse delays. Here, we use Stimulated Brillouin Scattering (SBS) to report the demonstration of on-chip slow, fast and negative group velocities with $\Delta \mathrm{n}_{\mathrm{g}}$ ranging from -44 to +130 , and delays of up to $23 \mathrm{~ns}$ at a pump power of $\sim 300 \mathrm{~mW}$ and propagation length of $7 \mathrm{~cm}$. These results are obtained using a highly-nonlinear chalocogenide $\left(\mathrm{As}_{2} \mathrm{~S}_{3}\right)$ rib waveguide, in which the confinement of both photons and phonons results in strong interaction. SBS can be used to achieve controllable pulse delays at room temperature over a large wavelength and signal-bandwidth [5]. These results open up a new set of photonic applications ranging from microwave photonics [8] to spectrometry [4].
\end{abstract}

Keywords: stimulated Brillouin scattering, on-chip, slow light, group index, chalocogenide, tunable delay

\section{INTRODUCTION}

Controlling the speed of a light pulse entails changing the group index $\mathrm{n}_{\mathrm{g}}$ : slow light, for which $\mathrm{n}_{\mathrm{g}}>1$, and fast light, for which $\mathrm{n}_{\mathrm{g}}<$ 1 , can be obtained by centering a pulse near a material $[2,3,5,7,9,10]$ or structural resonance $[1,6,11,12]$ where the induced change in dispersion gives rise to a large index slope [13]. The challenge in achieving on-chip slow and fast light is that the short interaction length requires a very large value of $\mathrm{n}_{\mathrm{g}}$ to achieve an appreciable pulse delay. Thus far, large values of group index have been obtained using resonances of atomic transitions [3, 7] or optical cavities [6,11,14] and multi-structured waveguides [1], however these techniques are fixed in frequency and/or limited in signal bandwidth, constraints which put severe restrictions on the maximum achievable pulse delay. In addition, the atomic transitions for which the largest values of $\mathrm{n}_{\mathrm{g}}$ are attained require specialized hardware, and have applications mainly in quantum information processing. An alternative approach for situations where integration or tunability (of wavelength, signal bandwidth and delay) is important involves Stimulated Brillouin Scattering (SBS), in which the photons interact coherently with phonons in an optical waveguide. Application of SBS on the chip-scale would provide a flexible, dynamically tunable system that can work at almost any wavelength of interest at room temperature and accommodating signal bandwidths covering $\mathrm{MHz}$ to tens of $\mathrm{GHz}$ [5]. However, the opto-acoustic interaction that drives the SBS process is typically extremely weak, leading to low values of $\mathrm{n}_{\mathrm{g}}$ for realistic pump powers, and so SBS experiments have invariably been performed in optical fibers several meters long. Application in a chip-scale device, which is important for the integration of delay components, requires at least a 10 -fold increase in the strength of the underlying interaction.

Here, we report on-chip control of the speed of a light pulse using SBS. We have observed slow ( $\sim 2307 \mathrm{~km} / \mathrm{s}$ ), fast and negative group velocities $\left(\mathrm{v}_{\mathrm{g}} \sim-6818 \mathrm{~km} / \mathrm{s}\right)$ in a $7 \mathrm{~cm}$ long chalcogenide $\left(\mathrm{As}_{2} \mathrm{~S}_{3}\right)$ rib waveguide, corresponding to group index changes ranging from $\sim-44$ to +130 with the largest $n_{g}$ achieved at a low gain of $\sim 23 \mathrm{~dB}$ [15]. To the best of our knowledge, this is the record $\mathrm{n}_{\mathrm{g}}$ in a SBS slow-light system and is 13 -fold larger than the previous value of $\mathrm{n}_{\mathrm{g}} \sim 10$ which was achieved in a $2 \mathrm{~m}$ long Erbium-

Nonlinear Optics and Applications VI, edited by Benjamin J. Eggleton, Alexander L Gaeta, Neil G. Broderick, Proc. of SPIE Vol. 8434, 84340O - (c) 2012 SPIE · CCC code: 0277-786X/12/\$18 · doi: 10.1117/12.923100 
doped Tellurite fiber at a gain of $\sim 53 \mathrm{~dB}$ [16]. We exploited the SBS process in our optical chip to tune the delay of a $25 \mathrm{~ns}$ long Gaussian pulse from $-7 \mathrm{~ns}$ to $\sim 22 \mathrm{~ns}$ (nearly one pulse width delay) while a $100 \mathrm{~ns}$ Gaussian pulse was delayed from $-6 \mathrm{~ns}$ to $23 \mathrm{~ns}$. The modification of the group index is large enough for us to directly measure it by tracking the phase of a narrow band signal. We present such measurements here for both slow and fast light regimes.

\section{ON-CHIP SBS SLOW AND FAST LIGHT}

To obtain slow light using SBS, a pump wave $\left(\omega_{\mathrm{p}}\right)$ must interact coherently with an acoustic wave at frequency $\left(\Omega_{\mathrm{B}}\right)$ and a counterpropagating wave at the Stokes frequency $\left(\omega_{\mathrm{s}}\right)$ (see Figure 1). A pulse in the counter-propagating wave then experiences a delay according to [17]

$$
\Delta T_{d}\left(\omega_{s}\right)=\frac{L_{e f f} \Delta n_{g}\left(\omega_{s}\right)}{c}=\frac{g_{B} I_{p} L_{e f f}}{\Lambda}
$$

where $\mathrm{L}_{\mathrm{eff}}$ is the effective length, $\mathrm{g}_{\mathrm{B}}$ is the Brillouin gain coefficient, $I_{D}$ is the pump intensity, and $\Gamma$ is the Brillouin linewidth. This delay can be tuned by changing the pump power, and fast light can be obtained by shifting the frequency of the signal to the anti-Stokes line. However, obtaining large values of $\Delta T_{d}$ on a chip, where $\mathrm{L}_{\text {eff }}$ is small, is difficult because the best values of $\Gamma$ for optical materials are on the order of tens of $\mathrm{MHz}$; this means that large delays require a very large $g_{B} I_{p}$ product. We approached this difficulty using a combination of materials and geometry. The Brillouin gain $g_{B}$ can be enhanced using high refractive index materials, provided other material parameters are comparable, because the coefficient of electrostriction scales with $\mathrm{n}^{8}$. Further enhancement can be gained by ensuring that the photons and phonons are both guided within the same structure. A material satisfying all the right conditions is chalcogenide glass, with refractive index $\mathrm{n}=2.4$ at $1550 \mathrm{~nm}$ and a longitudinal sound velocity of $\sim 2600 \mathrm{~m} / \mathrm{s}$ (compared to $\sim 5971 \mathrm{~m} / \mathrm{s}$ for silica). Chalcogenide glass has been shown to have both large Brillouin gain and Kerr nonlinear coefficients ( 100 times that of silica) [18-20], and the use of a microstructure waveguide, for which the mode area is small and hence $I_{p}$ is large, means that the power required to achieve the same delay in a given length is dramatically reduced.

Our device consisted of a $7 \mathrm{~cm}$-long chalcogenide $\left(\mathrm{As}_{2} \mathrm{~S}_{3}\right)$ rib waveguide on a silica substrate (see Figure 1). The optical chip used in the experiments has a cross-sectional area of $4 \mu \mathrm{m} \times 850$ $\mathrm{nm}, \mathrm{g}_{\mathrm{B}} \sim 0.74 \times 10^{-9} \mathrm{~m} / \mathrm{W}$ [21], $\mathrm{A}_{\mathrm{eff}} \sim 2.3 \mu \mathrm{m}^{2}$, Brillouin shift of $\sim 7.7 \mathrm{GHz}$ and linewidth of $\sim 36 \mathrm{MHz}$. Because of the large contrast in phase velocity of both sound and light between the waveguide and cladding, the acoustic and optical modes are both confined within the rib (Fig. 1b). We first measured the change in group index profile of the counter-propagating wave in the slow (fast) light regime. To do this we modulated the probe signal at the Stokes (anti-Stokes) frequency using a sinusoidal wave; the probe signal frequency was then detuned from centre of the Stokes (anti-Stokes) line while the coupled CW pump power was kept fixed. At each detuning, we measured the group delay of the sinusoidal signal and calculated the group index profile, shown in Fig. 2; good agreement was found with theoretical calculations using a Lorentzian model for the interaction. In the slow-light regime, we observed a maximum group index change of $\sim 68$, implying a reduction of the group velocity of light to $4411 \mathrm{~km} / \mathrm{s}$ at a pump power of 
$152 \mathrm{~mW}$. For a signal centered at $\omega$ as the slope of $\mathrm{n}(\omega)$ is negative (see Fig. 1), leading ultimately to negative values of $\mathrm{n}_{\mathrm{g}}$. We use the negative change in group index to demonstrate fast and negative group velocities: a group index change of $\Delta \mathrm{n}_{\mathrm{g}} \sim-44$ was measured at a power of $\sim 91 \mathrm{~mW}$, resulting in a negative group velocity $(\sim-6818 \mathrm{~km} / \mathrm{s})$. This implies that the body of the pulse exits the medium before entering it, causality being preserved because the information in the pulse is carried by the signal velocity.
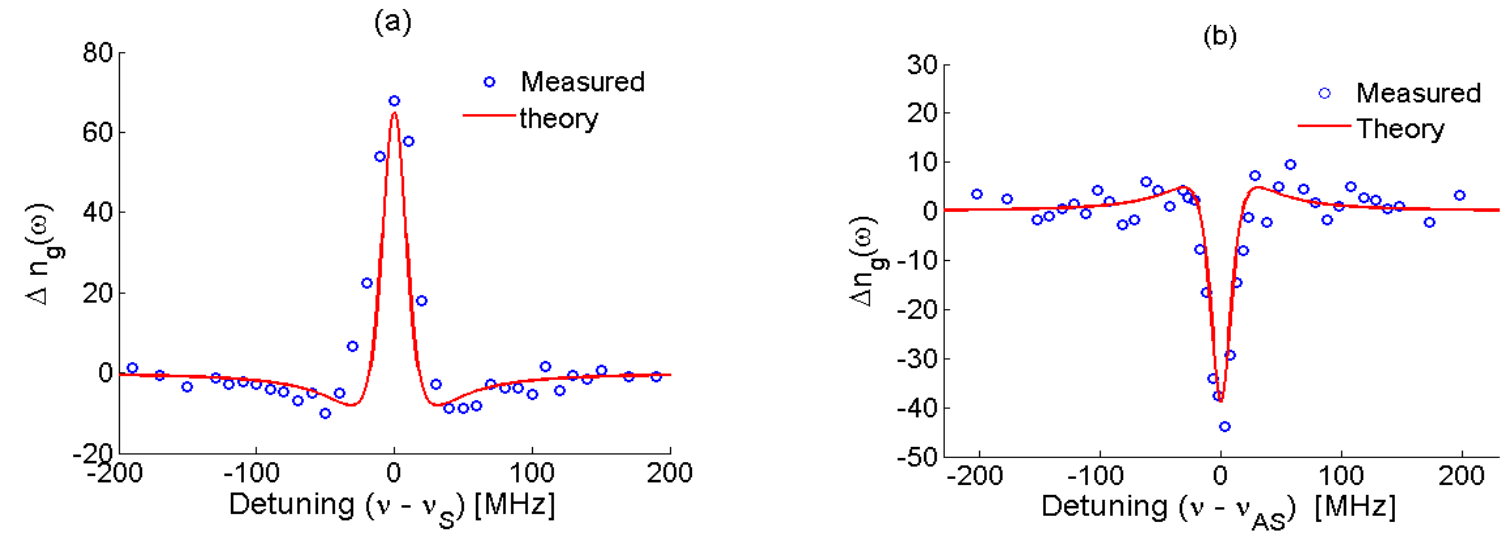

Fig 2. Calculated (solid) and measured (circles) group-index profile for (a) slow light, and (b) fast light. A sinusoidal modulation of $10 \mathrm{MHz}$ for slow-light and $3 \mathrm{MHz}$ for fast light was imposed on the probe carrier wave whose frequency was detuned from the Stokes (anti-Stokes) for slow (fast) light. The coupled CW pump power of $\sim 152 \mathrm{~mW}$ for slow light and $\sim 91 \mathrm{~mW}$ for fast light were used.

(a)

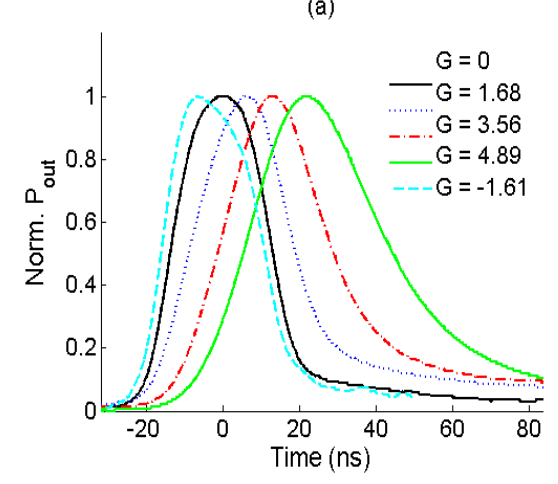

(b)

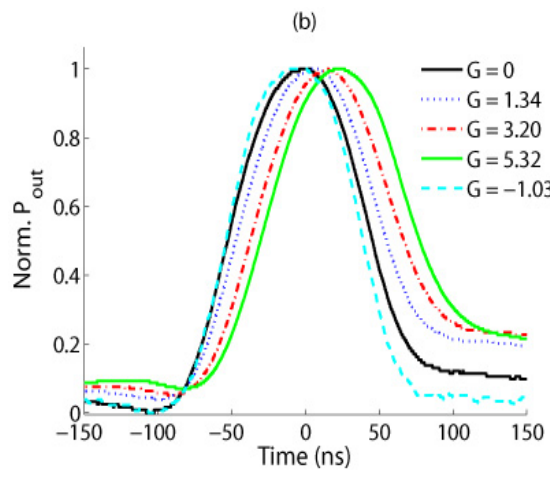

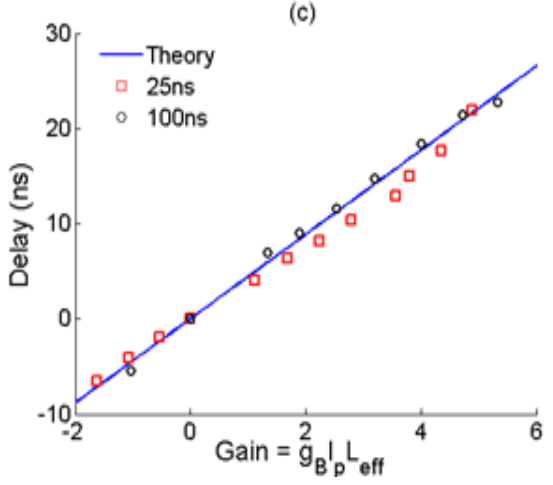

Fig 3. Measured output pulses for different gain values demonstrating SBS induced slow- and fast light for (a) $25 \mathrm{~ns}$ Gaussian pulse (b) 100 ns Gaussian pulse and (c) measured and theoretical delay, obtained using Eq. (1), vs. gain.

We then measured the delays directly by modulating the counter-propagating signal with a Gaussian pulse. For these experiments we used a pulsed pump to increase the on-chip peak pump power to $\sim 300 \mathrm{~mW}$. Pump pulses were $1 \mu$ s long with a duty cycle of $20 \%$ and were synchronized with the probe pulses. Figures 3(a) and 3(b) show the delay for pulse-widths of $\sim 25 \mathrm{~ns}$ and $\sim 100 \mathrm{~ns}$, respectively, for different values of gain $\left(G=g_{B} I_{p} L_{\text {eff }}\right)$. As the pump power was increased (decreased) the group index increased (decreased) according to Eq. (1) and the speed of light pulse was reduced (advanced) for pulses centered at $\omega$ s ( $\omega$ as) resulting in slowand fast-light. For a $25 \mathrm{~ns}$ long Gaussian pulse, we achieved a delay of $\sim 22 \mathrm{~ns}$ at a peak pump power of $\sim 300 \mathrm{~mW}$ whilst for a $100 \mathrm{~ns}$ long Gaussian pulse the maximum delay was $\sim 23$ ns. From the measured slow light delay for a 100 ns pulse we calculated the maximum achieved $n_{g}$ to be $\sim 130$, which was approximately twice the group index measured at the Stokes shift from group delay 
measurements at $\sim 152 \mathrm{~mW}$ (see Fig. 2) and implies that the speed of light was 130 times smaller than that in vacuum. This is the largest reduction ever achieved in the speed of light pulses using SBS.

For fast light pulse-delay experiments we only use a CW pump. For a $100 \mathrm{~ns}$ pulse, a maximum pulse advance of $\sim-6 \mathrm{~ns}$ was obtained at $\mathrm{G} \sim-1.03$. The output pulses for the $25 \mathrm{~ns}$ probe pulse exhibit significant broadening for large gain values as the different frequency components experienced different delay and gain resulting in pulse distortion. For the $100 \mathrm{~ns}$ probe pulse there was almost no distortion; the output pulses were nearly the same width as the input pulse. Figure 3(c) shows a comparison of our measured delay with the theoretical delay calculated using Eq. (1) showing a good agreement between theory and experiments for $100 \mathrm{~ns}$ pulse while the slow-light delay for a $25 \mathrm{~ns}$ pulse was slightly smaller as would be expected.

\section{CONCLUSION}

In conclusion, we have demonstrated on-chip slow, fast and negative group velocities using SBS where $\Delta \mathrm{n}_{\mathrm{g}}$ was continuously varied from -44 to +130 resulting in group velocities of $2307 \mathrm{~km} / \mathrm{s}$ and $-6818 \mathrm{~km} / \mathrm{s}$ for slow- and fast-light respectively. Such a wide variation allowed continuously tunable, on-chip delays ranging from $-6 \mathrm{~ns}$ to $+23 \mathrm{~ns}$ in a $7 \mathrm{~cm}$ long photonic chip with the maximum delay achieved at the relatively low pump power of $\sim 300 \mathrm{~mW}$. The importance of this demonstration is that it opens up the SBS process to the scalability of on-chip devices: for applications such as true-time delay for phased array antenna and microwave filters where multiple delay lines with tunable delays of 1-10 ns are required to control multiple Radar elements, the integration and operation flexibility afforded by on-chip slow and fast light is highly desirable [8]. Furthermore, the large dispersion offers increased sensitivity for ultra-compact spectrometers [4]. The ability to control light speed on a chip using the phonon modes leads not only to these new applications and technologies, but also provides a new platform for exploring fundamental interactions between sound and light.

\section{ACKNOWLEDGEMENTS}

This work was supported by the Australian Research Council (ARC) through its Discovery grant (DP1096838), ARC Federation fellowship and Centre of Excellence scheme (CUDOS) and in part by the US Department of Defense through AFOSR/AOARD grant (grant \# FA23861114030).

\section{REFERENCES}

1. $\quad$ Baba, T., Slow light in photonic crystals. Nature Photonics, 2008. 2(8): p. 465-473.

2. $\quad$ Boyd, R.W. and D.J. Gauthier, Controlling the Velocity of Light Pulses. Science, 2009. 326(5956): p. 10741077.

3. Hau, L.V., et al., Light speed reduction to 17 metres per second in an ultracold atomic gas. Nature, 1999. 397(6720): p. 594-598.

4. Sternklar, S., et al., Mutually-modulated cross-gain modulation and slow light. Journal of Optics, 2010. 12(10).

5. Thevenaz, L., Slow and fast light in optical fibres. Nature Photonics, 2008. 2(8): p. 474-481.

6. Vlasov, Y.A., et al., Active control of slow light on a chip with photonic crystal waveguides. Nature, 2005. 438(7064): p. 65-69.

7. Wu, B., et al., Slow light on a chip via atomic quantum state control. Nature Photonics, 2010. 4(11): p. 776-779.

8. Chin, S., et al., Broadband true time delay for microwave signal processing, using slow light based on stimulated Brillouin scattering in optical fibers. Optics Express, 2010. 18(21): p. 22599-22613.

9. $\quad$ Okawachi, Y., et al., Tunable all-optical delays via Brillouin slow light in an optical fiber. Physical Review Letters, 2005. 94(15).

10. Okawachi, Y., et al., All-optical slow-light on a photonic chip. Optics Express, 2006. 14(6): p. 2317-2322.

11. Mok, J.T., et al., Dispersionless slow light with 5-pulse-width delay in fibre Bragg grating. Electronics Letters, 2007. 43(25): p. 1418-1419. 
12. Poon, J.K.S., et al., Slowing light with Fabry-Perot resonator arrays. Journal of the Optical Society of America B-Optical Physics, 2007. 24(11): p. 2763-2769.

13. Jackson, J.D., Classical Electrodynamics. 2001, John Wiley \& Sons.

14. Lee, M.J., et al., SBS gain-based slow-light system with a Fabry-Perot resonator. Optics Communications, 2008. 281(10): p. 2975-2984.

15. Pant, R., et al., Photonic chip based tunbale slow and fast light via stimulated Brillouin scattering. Optics Letters, 2012.37 (5): p. 969-971.

16. Abedin, K.S., G.W. Lu, and T. Miyazaki, Slow light generation in singlemode Er-doped tellurite fibre. Electronics Letters, 2008. 44(1): p. 16-U21.

17. Zhu, Z.M. and D.J. Gauthier, Numerical study of all-optical slow-light delays via stimulated Brillouin scattering in an optical fiber. Journal of the Optical Society of America B-Optical Physics, 2005. 22(11): p. 2378-2384.

18. Abedin, K.S., Brillouin amplification and lasing in a single-mode AS(2)Se(3) chalcogenide fiber. Optics Letters, 2006. 31(11): p. 1615-1617.

19. Eggleton, B.J., B. Luther-Davies, and K. Richardson, Chalcogenide Photonics. Nature Photonics, 2011. 5: p. 141-148.

20. Eggleton, B.J., et al., Photonic chip based ultrafast optical processing based on high nonlinearity dispersion engineered chalcogenide waveguides. Laser \& Photonics Reviews, 2012. 6(1): p. 97-114.

21. Pant, R., et al., On-chip stimulated Brillouin scattering. Optics Express, 2011. 19(9): p. 8285-8290. 